\title{
Accuracy Investigation of Dual Mode Markers for Navigated Dental Implant Surgery with a new 3d Realtime Navigation System DENACAM
}

\author{
Simone Hemm ${ }^{1}$, Stephan Böhringer ${ }^{2}$, Quentin Garnier ${ }^{1}$, Michael \\ Breitenstein $^{2}$, Claudio Minonzioํㅜ, Frank Berlinghoff², Philipp Jürgens ${ }^{2,3}$ \\ and Erik Schkommodau ${ }^{1}$ \\ ${ }^{1}$ Institute for Medical and Analytical Technologies, University of Applied Sciences and Arts \\ Northwestern Switzerland, Muttenz, 4132, Switzerland, \\ ${ }^{2}$ Mininavident AG, Basel, 4057, Switzerland \\ ${ }^{3}$ Hightech Research Center of Cranio-Maxillofacial Surgery, University Hospital, Basel, 4031, \\ Switzerland \\ simone.hemm@fhnw.ch
}

\begin{abstract}
Dental implants are in 96\% positioned free hand including a risk of suboptimal placement. A miniaturized navigation system has been developed to overcome these limitations by fixing two stereo cameras to the drill to guide the surgeon during implant insertion. The aim of the present study was to develop and analyse a dual-mode marker with a highly precise, high contrast optical pattern, visible by the camera system, on a precise substrate clearly identifiable on Cone Beam CT (CBCT) images and sterilisable. The marker was developed with a laser engraved optical pattern brought to a ceramic substrate containing two holes to ensure clear identification of the position of the marker within CBCT images.

The substrate dimensions were verified with a micrometer gauge and compared to the expected tolerances. The position and angular error of the optical pattern on the substrate was analysed on optical microscope images. Six markers were exposed to 20 cleaning and steam sterilization cycles and changes were analysed on optical and scanning electron microscope (SEM) images. Energy dispersive X-ray spectroscopy (EDX) analysis was performed to study the percentage composition of the different elements.

Substrate dimensions and angular errors remained always within the defined tolerances. Positioning errors were higher than the tolerances for some of the measurements. The error distribution indicates a systematic error. Results were reproducible over the samples. No changes could be observed visually on the optical
\end{abstract}


microscope images between the initial marker and the $20^{\text {th }}$ sterilization cycle. EDX results show slight percentage variations of the different elements between the different cycles. Fluctuations were most important for the carbon-content.

A dual mode marker could be developed with a very accurate optical pattern very precisely positioned on a ceramic substrate making any marker calibration procedure unnecessary. Precision analysis confirmed a reproducible position of the pattern on the substrate, indicating a stable and robust process. Systematic errors can be corrected directly at the used Laser system. Cleaning and sterilization tests confirmed the stability of the dual-mode marker after cleaning and sterilization. The present study has shown that highly accurate, high contrast and robust markers can be manufactured using laserengraving technology on ceramic substrates.

\section{Introduction}

Dental implants are in $96 \%$ positioned free hand including a risk of suboptimal placement like drilling in wrong angle or hitting a nerve canal. Different approaches exist to limit such error sources. One are drill guides, which have been reported to produce apical deviations between 0.6 up to $4.5 \mathrm{~mm}$ (D'Haese, 2012) (Wittneben, 2014). Computer-guided and navigated implant surgery solutions are coming more and more on the market (Gulati, 2015).

A miniaturized navigation system has been developed to overcome these limitations. Two cameras are fixed on the drill to guide the surgeon during implant insertion. The procedure is planned on Cone Beam CT (CBCT) images and the planning is then transferred to the navigation software. To be able to follow the current drill position online on the planned images, a dual mode marker had to be developed, i.e. with a highly precise, high contrast optical pattern, visible by the camera system, on a precise substrate clearly identifiable on CBCT images and sterilisable. The aim of the present work was to develop such a marker and to verify accuracy and sterilisability.

\section{Materials and Methods}

A dual mode marker was developed with a laser engraved optical pattern composed of rectangles and circles brought to a ceramic substrate of $10 \times 15 \mathrm{~mm}$ in size (Figure 1). High contrast could be guaranteed by using a specific PET-film. Two holes in the ceramic substrate ensure the clear identification of the position of the marker within the CBCT images.

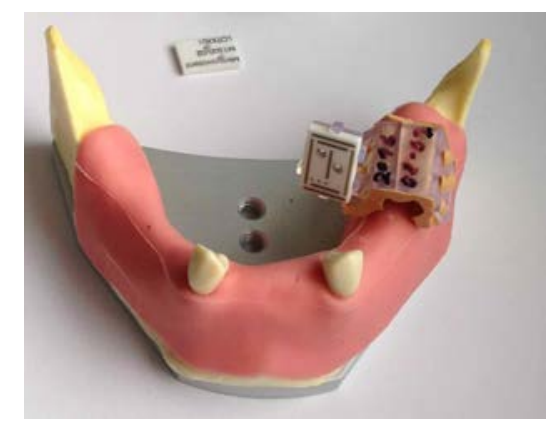

Figure 1: Developed marker fixed on an impression tray to a lower jaw model 
The substrate dimensions were verified with a micrometer gauge and compared to the expected tolerances (general tolerance: $0.05 \mathrm{~mm}$; thickness tolerance: $0.1 \mathrm{~mm}$ ). The position of the optical pattern on the substrate was analysed based on optical microscope images (Olympus BX61). Binary images were generated to identify position and direction of the rectangles. The rotational and the position errors of the optical pattern on the substrate were determined by matching the binary image with an artificial picture of the theoretical marker, and they were compared to the given tolerances (general tolerance: $\pm 0.05 \mathrm{~mm}$; angular tolerance: \pm 0.25 degree). Six markers were exposed to 20 cleaning (deconex Power Zyme, Bohrer AG) and sterilization (autoclave, $134^{\circ} \mathrm{C}, 18 \mathrm{~min} ; 18 \mathrm{~min}$ drying) cycles. Optical microscopic images (under standardized light conditions) and scanning electron microscope (SEM) images were taken at $0,5,10$ and 20 cycles and compared. In addition, energy dispersive X-ray spectroscopy (EDX) analysis was performed to study the percentage composition of the different elements.

\section{Results}

\subsection{Precision analysis}

Substrate dimensions remained always within the defined tolerances. Figure 2 shows results of the precision analysis of the optical pattern on the substrate. Angular errors remained always below the given tolerances while positioning errors were higher than the tolerances for some of the measurements with a maximum of $0.12 \mathrm{~mm}$. The error distribution indicates that it is probably a systematic error. Results were reproducible over the samples. 

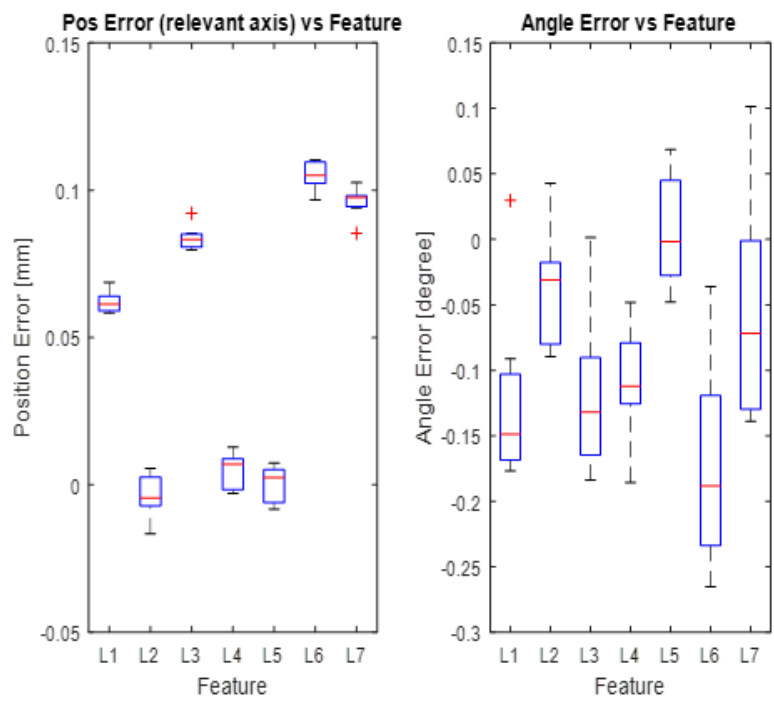

Figure 2: Position error and angular error for the different features L1 to L7 (different rectangles and circles of the pattern) of the optical pattern in comparison to their theoretical position on the substrate.

\subsection{Cleaning and Sterilization Tests}

No changes could be observed visually on the optical microscope images between the initial marker and the $20^{\text {th }}$ sterilization cycle. EDX results show slight percentage variations of the different elements between the different cycles. Fluctuations were most important for the carbon-content ( $28.6 \% \pm 8.0 \%$ ) and less for titanium $(4.0 \% \pm 0.7 \%)$, present in the laser-engraved optical pattern.

\section{Discussion}

A dual mode marker could be developed with a very accurate optical pattern very precisely positioned on a ceramic substrate making any marker calibration procedure unnecessary.

Precision analysis confirmed a reproducible position of the pattern on the substrate during the same engraving cycle, indication for a stable and robust process. Due to changing conditions of the laser engraving for example depending on the running time of the laser, the current deviations from the theoretical position will be determined at the beginning of each process, transformed to a correction factor and injected in the laser engraving machine/device.

Cleaning and sterilization tests showed no visual deterioration or contrast change of the laser engraved optical pattern so that it can be assumed that much more cycles can be performed in practice. The percentage variation of the elements on the surface are due to the fact that carbon can be deposited on the surface during cleaning or sterilisation and that if the concentration of one element changes, all the others change in the opposite direction. Higher variations in titanium would have been expected in case of a delamination of the optical pattern inducing visible contrast changes.

Other coating procedures of ceramic markers are possible such as Diamond-like-Carbon coatings (Hemm, 2014), but the present study has shown that a high accuracy can be obtained with laserengraving with the help of a contrast enhancing PET-film. 
First tests of the overall tracking precision of the system within its whole working room have confirmed the expected overall submillimetric precision of the system (Böhringer, 2016).

\section{References}

\section{Literaturverzeichnis}

Böhringer, S. (2016). Accuracy investigation of a new mininavigation system for dental implants. Biomed Tech 61(s1), S115.

D'Haese, J. (2012). Accuracy and complications using computer-designed stereo-lithographic surgical guides for oral rehabilitation by means of dental implants: a review of the literature. Clin Implant Dent Relat Res 14(3),, 321-33.

Gulati, M. (2015). Computerized implant-dentistry: Advances toward automation. J Indi-an Soc Periodontol, 5-10.

Hemm, S. (2014). Diamond-like carbon coating for highly precise and biocompatible optical markers for surgical navigation. Biomedizinische Technik, 48th DGBMT Annual Conference, (S. DOI: 10.1515/bmt-2014-4187).

Wittneben, J.-G. (2014). Complication and Failure Rates with Implant-Supported Fixed Dental Prostheses and Single Crowns: A 10-Year Retrospective Study. Clin Implant Dent Res, 356364.

\section{Disclosures}

F. Berlinghoff is CEO, M. Breitenstein Quality and Product Manager, S. Böhringer responsible for R\&D, P. Jürgens CMO and E. Schkommodau co-founder at Mininavident AG. The project was a collaborative project between Mininavident AG, FHNW and the University Hospital in Basle, financed by the Swiss Commission for Technology and Innovation. 